Gab es doch eine Alternative zur restriktiven Finanz- und Haushaltspolitik der von Heinrich Brüning geführten Präsidialkabinette? Hätte Weimars Ende vielleicht verhindert werden können, wenn der Reichskanzler nicht so stur gewesen wäre und ein französisches Kreditangebot akzeptiert hätte? Paul Köppen bejahte diese Fragen in der Juliausgabe 2014 der Vierteljahrshefte für Zeitgeschichte. Knut Borchardt, einer der international angesehensten deutschen Wirtschaftshistoriker, entzieht Köppens Thesen die Grundlage; er weist nach, dass es ein französisches Kreditangebot nicht gegeben hat.

Knut Borchardt

\title{
Eine Alternative zu Brünings Sparkurs?
}

Zu Paul Köppens Erfindung französischer Kreditangebote

\section{I.}

In der nun schon mehr als dreißig Jahre andauernden Debatte über Zwangslagen und Handlungsspielräume der deutschen Wirtschaftspolitik in der Weltwirtschaftskrise der frühen dreißiger Jahre ${ }^{1}$ sind ausgiebig denkbare Alternativen zum Sparkurs der Regierung Brüning diskutiert worden. Vielleicht hätte das Schicksal der Weimarer Republik gewendet, Hitler vermieden werden können? Dabei herrschte alsbald Einvernehmen darüber, dass das Reich seine regelmäßig auflaufenden Haushaltsdefizite nicht mehr - wie in der Aufschwungsperiode 1924-1929 - nach Bedarf mit Krediten habe abdecken können und dass die Regierung deshalb zum Sparkurs mit seiner deflatorischen Wirkung gezwungen gewesen $\mathrm{sei}^{2}$.

\footnotetext{
${ }^{1}$ Ausgelöst wurde sie durch meinen Vortrag „Zwangslagen und Handlungsspielräume in der großen Wirtschaftskrise der frühen dreißiger Jahre. Zur Revision des überlieferten Geschichtsbildes“, in: Bayerische Akademie der Wissenschaften, Jahrbuch 1979, München 1979, S. 87-132; wieder abgedruckt u. a. in: Knut Borchardt, Wachstum, Krisen, Handlungsspielräume der Wirtschaftspolitik. Studien zur Wirtschaftsgeschichte des 19. und 20. Jahrhunderts, Göttingen 1982, S.165-182 u. S. 265-283; englische Fassung in: Knut Borchardt, Perspectives on Modern German Economic History and Policy, Cambridge 1991, S. 143-160 u. S. 238-258. In der von Paul Köppen verwendeten Fassung, in: Michael Stürmer (Hrsg.), Die Weimarer Republik. Belagerte Civitas, Königstein/Ts. 1980, S. 318-338, ist der Anmerkungsteil erheblich verkürzt, worauf dort ausdrücklich hingewiesen wird.

${ }^{2}$ Zur Tatsache und den Gründen fehlender Kredite vgl. u.a. Harold James, The Reichsbank and Public Finance in Germany 1924-1933. A Study of the Politics of Economics during the Great Depression, Frankfurt a. M. 1985, S. 95-135; Theo Balderston, The Origin and Course of the German Economic Crisis. November 1923 to May 1932, Berlin 1993; Ursula Bachmann, Reichskasse und öffentlicher Kredit in der Weimarer Republik 1924-1932, Frankfurt a. M. u.a. 1996; Albrecht Ritschl, Reparation transfers, the Borchardt hypothesis, and the Great Depression in Germany 1929-1932: a guided tour for hard-headed Keynesians, in: European Review of Economic History 2 (1998), S.49-72; ders., Deutschlands Krise und Konjunktur
} 
Umso überraschender ist die Veröffentlichung des Artikels „'Aus der Krankheit konnten wir unsere Waffe machen. ' Heinrich Brünings Spardiktat und die Ablehnung der französischen Kreditangebote 1930/31"von Paul Köppen in dieser Zeitschrift ${ }^{3}$. Schon in der vorangestellten Zusammenfassung wird der Eindruck erweckt, es habe die Möglichkeit eines anderen Kurses, eine „Alternative“ gegeben. Köppen meint, in den Akten den Beweis für Kreditangebote von französischer Seite gefunden zu haben. Brüning sei nur nicht darauf eingegangen. In seinem Aufsatz befasst sich der Autor dann vor allem mit der Frage, warum Brüning die Angebote nicht angenommen hat. Er kommt zum Ergebnis, dass Brünings restriktive Finanzpolitik „weniger mit Zwangslagen als mit Taktik zu tun“ hatte ${ }^{4}$. Das führt die Diskussion auf den Stand vor der Eröffnung der sogenannten „BorchardtKontroverse" zurück ${ }^{5}$.

Im Folgenden soll es vornehmlich um die Behauptung gehen, von der alles Weitere bei Köppen abhängt: Es habe französische Kreditangebote gegeben, die es Brüning möglich gemacht hätten, auf seine restriktive Finanz-, insbesondere Haushaltspolitik zu verzichten und somit „die sozialen und ökonomischen Folgen der Weltwirtschaftskrise zumindest abzumildern“, wie es in der Zusammenfassung heißt ${ }^{6}$. Es ist leider nicht der Platz, hier auch nachzuholen, was Köppen versäumt, nämlich die Geschichte der realen Gründe für die Zwangslage in den Blick zu nehmen, also die Ursachen und den Charakter der finanzwirtschaftlichen Probleme. Darauf müsste sich aber jemand einlassen, der von einer Alternative spricht. Freilich, Köppen gibt am Ende zu, dass es „zu einem guten Stück spekulativ“ bleibt, „ob und inwieweit die französischen Kredite den Gang der Geschichte hätten ändern können“. Doch steht für ihn fest: „Es war nicht zuletzt die frühe Festlegung Brünings auf sein finanzpolitisches Krisenkonzept, die in Berlin eine gründlichere Abwägung der französischen Stabilisierungsbemühungen verhinderte. “7

Merkwürdigerweise wird in der dem Text vorangestellten Zusammenfassung von „Kreditangeboten auf dem Höhepunkt der Krise“ gesprochen. Dabei behandelt Köppen doch ausdrücklich französisch-deutsche Kontakte in der Anfangszeit der Regierung Brüning von Juli 1930 bis März 1931². Damals steckte Deutschland zwar schon seit einem Jahr in einer Schuldenkrise, aber noch niemand hat die

1924-1934. Binnenkonjunktur, Auslandsverschuldung und Reparationsproblem zwischen Dawes-Plan und Transfersperre, Berlin 2002; ders., Reparations, Deficits, and Debt Default: The Great Depression in Germany, in: Nicholas Crafts/Peter Fearon, The Great Depression of the 1930s. Lessons for Today, Oxford 2013, S. 110-139.

${ }^{3}$ VfZ 62 (2014), S. 349-375.

${ }^{4}$ Ebenda, S. 355 .

${ }^{5}$ Dies geschieht anscheinend absichtlich, denn Köppen beendet seinen Aufsatz mit dem Hinweis auf den kritischen Artikel von Carl-Ludwig Holtfrerich, Alternativen zu Brünings Wirtschaftspolitik in der Weltwirtschaftskrise?, in: Historische Zeitschrift 235 (1982), S. 605-631, mit dem 1982 die Kontroverse eröffnet worden ist.

${ }^{6}$ Köppen, Brünings Spardiktat, S. 349.

${ }^{7}$ Ebenda, S. 372.

${ }^{8}$ Insofern ist ihm zuzustimmen, dass in dieser Zeit die Haushaltsnöte hätten behoben werden müssen, wenn überhaupt an eine Änderung der von der Vorgängerregierung ererbten Sparpolitik zu denken gewesen wäre. 
Dramatik des weiteren Krisenverlaufs geahnt. „Auf dem [späteren] Höhepunkt der Krise“, nämlich in der Banken- und Währungskrise im Juli 1931, hat es tatsächlich ein Kreditangebot Frankreichs gegeben. Auf dieses geht Köppen auf den letzten zwei Seiten ein, ohne auch nur näherungsweise den so ganz anderen Kontext - und den erreichten Forschungsstand - zu beachten. Weil die französische Idee einer internationalen Anleihe nicht dem Zweck hätte dienen können, Brüning einen weniger deflationären Kurs zu ermöglichen, kann sie in der Diskussion darüber, ob es eine Alternative zu Brünings Sparpolitik gegeben habe, außer Betracht bleiben ${ }^{9}$.

\section{II.}

Wenn von Kreditangeboten die Rede ist, die Brüning einen weniger deflationären Kurs hätten ermöglichen sollen, erwartet man (zumindest der Ökonom) unter anderem Antwort auf folgende Fragen:

1. Wer sollte das Kapital geben, die französische Regierung bzw. das Parlament, französische Banken oder das französische Publikum als Zeichner von öffentlich aufgelegten Anleihen?

2. Wer war als Empfänger solcher Gelder gedacht? Die Reichsregierung und/ oder mit deren Haushalt verbundene Länder, Gemeinden, Unternehmen in öffentlicher oder privater Hand?

3. Um welche Summen könnte es gegangen sein? Angesichts der erheblichen Lücken in der Deckung der Haushalte konnten nur hohe Beträge helfen.

4. An welche Laufzeiten hat man gedacht? So wie die Dinge lagen, war kurzfristiger Kredit in Hinblick auf einen erwünschten Strategiewechsel keine Lösung. Wenn aber längerfristig, wie lange?

Angesichts der Bedeutung der Behauptung von französischen Kreditangeboten ist es verwunderlich, dass sich Köppen in seinem Aufsatz mit keiner dieser Fragen befasst hat. Vielmehr stützt er sich auf ein einziges Dokument, den Bericht des deutschen Botschafters in Paris über ein 2 1⁄2 stündiges Gespräch am 9. Juli 1930 mit Ministerpräsident Tardieu, Außenminister Briand und dem Generalsekretär des Außenministeriums Berthelot ${ }^{10}$.

\footnotetext{
${ }^{9}$ Köppen übersieht hier auch die Ablehnung der französischen Idee durch die Regierungen und Notenbanken der USA und Englands. Vgl. u.a. Edward W. Bennett, Germany and the Diplomacy of the Financial Crisis, Cambridge/MA 1962. Statt vieler Belege aus den Akten vgl. Vermerk des Staatssekretärs Pünder über eine Unterredung des Reichskanzlers mit dem amerikanischen Botschafter Sackett, 29.7.1931, in: Akten der Reichskanzlei. Die Kabinette Brüning I u. II, hier Bd. 2, bearb. von Tilman Koops, Boppard 1982, S. $1453 \mathrm{f}$.

${ }^{10}$ Vgl. Telegramm des Botschafters in Paris von Hoesch an das Auswärtige Amt v. 9. 7. 1930, in: Akten zur deutschen auswärtigen Politik 1918-1945 (ADAP), Serie B: 1925-1933, bearb. von Peter Grupp u. a., Göttingen 1980, hier Bd. XV: 1. Mai bis 30. September 1930, Nr. 129, S. 312-315. Nachfolgend wird bei Zitaten aus den ADAP der Hinweis auf die Serie fortgelassen. Dieses Dokument ist in der Forschung schon vielfach zitiert worden, ohne dass ein Kreditangebot erkannt worden wäre.
} 
Leider ist es nötig, genauer darauf einzugehen, was Köppen über den Inhalt des Gesprächs schreibt, und dies mit dem Originaltext zu vergleichen. Zu dem hier interessierenden Teil des Botschafterberichts schreibt Köppen:

\begin{abstract}
„Zum einen äußerten der französische Ministerpräsident André Tardieu, sein Außenminister Briand und dessen Staatssekretär Philippe Berthelot etwas überraschend ihr aufrichtiges Verständnis für die neue deutsche Rhetorik, weil man die Notwendigkeit anerkannte, dass Kanzler Brüning auf die ,Stimmung im deutschen Volk Rücksicht zu nehmen' habe, zum anderen sprachen sie unerwartet über Geld: Wie von Hoesch sofort nach Berlin telegrafierte, wies Tardieu darauf hin, dass augenblicklich etwa 25 Milliarden Franken kurzfristiger Kredite in Deutschland umliefen, und ausdrückte Bedauern, daß diese Kredite ohne Oberaufsicht und Regelung durch die beiderseitigen Regierungen normalerweise ihren Weg über die Schweiz und Holland nähmen, wobei die Zwischenhändler große Gewinne einsteckten, während Frankreich geringe Zinsen vergütet erhalte und Deutschland hohe Zinsen zahlen müsse. Folgerichtig offerierte die französische Seite eine ,direkte Verständigung zwischen den Regierungen unter Beiziehung von Sachverständigen über direkte Gewährung solcher kurzfristigen Kredite von französischen Banken an deutsche Geldinstitute."11
\end{abstract}

Ohne sich näher mit dem Inhalt und der Bedeutung des Berichteten zu befassen, nennt Köppen dies im Folgenden eine „Finanzspritze“, ein „Angebot“, „eine französische Finanzhilfe“, eine „französische Kreditofferte“12. Wiederholt ist sogar von „Kreditangeboten“ im Plural die Rede.

Vergleicht man Köppens Wiedergabe mit dem in den Akten zur Deutschen Auswärtigen Politik abgedruckten Original, fallen zwei Dinge ins Auge: Erstens die tendenziöse Unvollständigkeit des Zitierten gegenüber dem von Hoesch an das Auswärtige Amt Telegraphierten und zweitens das Falsche der Behauptung, es habe sich hier um ein Kreditangebot gehandelt. Zum Beweis hier der vollständige Text:

Es sei unbedingt nötig, „daß von Deutschland jetzt im Anschluß Räumungsvorgangs irgendein Auftakt im Sinne einer Anerkennung der französischen Loyalität und im Sinne eines Bekenntnisses zu der von Stresemann und Briand eingeleiteten und von Tardieu später mit aufgenommenen Verständigungspolitik erklingt. Alsdann werde der Weg offen sein, sich praktischen Fragen zuzuwenden.

Als solche Fragen erwähnte Tardieu zunächst das Gebiet der Kreditgewährung an Deutschland. Er hinwies darauf, daß augenblicklich etwa 25 Milliarden Franken kurzfristiger Kredite in Deutschland umliefen, und ausdrückte Bedauern, daß diese Kredite ohne Oberaufsicht und Regelung durch die beiderseitigen Regierungen normalerweise ihren Weg über die Schweiz und Holland nähmen, wobei die Zwischenhändler große Gewinne einsteckten, während Frankreich geringe

${ }^{11}$ Köppen, Brünings Spardiktat, S. $351 \mathrm{f}$.

12 Ebenda, u.a. S. 352 u. S. 354. 


\begin{abstract}
Zinsen vergütet erhalte und Deutschland hohe Zinsen zahlen müsse. Er anregte direkte Verständigung zwischen den Regierungen unter Beiziehung von Sachverständigen über direkte Gewährung solcher kurzfristigen Kredite von französischen Banken an deutsche Geldinstitute. Mein Hinweis darauf, daß für uns vor allem die Gewährung langfristiger Kredite interessant wäre, begegnete einer ziemlich zurückhaltenden Aufnahme. "13
\end{abstract}

Es verdient meiner Meinung nach durchaus Beachtung, dass Tardieu den Beginn von Erörterungen zu dieser wie den anderen im Bericht von Hoesch genannten „praktischen Fragen“von Voraussetzungen abhängig gemacht hat, die nicht leicht und ganz sicher nicht umgehend zu erfüllen waren. „Alsdann“ kann ja nicht heißen: „schon jetzt“. Was sodann den Inhalt betrifft, hat Tardieu gewiss nichts angeboten, was Brüning in seinen Finanznöten hätte helfen können. Es ging dem Ministerpräsidenten allein um Kredite von und an Banken. Für die französische Regierung war es offensichtlich ein Ärgernis, dass französische Banken Kredite an in der Schweiz und in Holland domizilierende Banken (darunter auch solche in deutschem Besitz) gaben, die dann ihrerseits aus den erhaltenen Mitteln Kredite an Institute in Deutschland gewährten. Das gestaltete es für die französische Regierung schwieriger, den Verkehr politisch zu steuern. Gerade dieser Umstand machte aber den Charme für die deutsche Seite aus. Sie konnte deshalb keinerlei Interesse an dem französischen Vorschlag haben, sich an einer Kontrolle zu beteiligen - selbst dann nicht, wenn damit eine (kaum zu kalkulierende) Chance für mehr Bankkredit aus Frankreich verbunden gewesen sein sollte ${ }^{14}$.

Weil er die Sache so gänzlich missversteht, kann sich Köppen darüber beklagen, dass Berlin erst spät und dann „mit auffallendem Desinteresse“15 reagiert habe. Dabei hätte ihn eine genaue Lektüre des Erlasses von Staatssekretär von Bülow vom 7. August dazu bringen können, die Sorgfalt der Vorbereitung der Antwort und das Vernünftige, ja das Wegweisende in ihr zu erkennen ${ }^{16}$. Bülow hat nämlich neben guten Gründen dafür, der französischen Idee einer Regulierung

\footnotetext{
${ }^{13}$ Hoesch an Auswärtiges Amt v. 9.7.1930, in: ADAP, Bd. XV, Nr. 129, S. 313 (Hervorhebungen durch den Verfasser).

${ }^{14}$ James, The Reichsbank, S. 124, hat die Vermutung geäußert, Tardieu hätte die 25 Mrd. Francs $=4,1 \mathrm{Mrd}$. RM als Drohpotenzial genannt, was wohl nicht von der Hand zu weisen ist. Franz Knipping, Deutschland, Frankreich und das Ende der Locarno-Ära 1928-1931. Studien zur internationalen Politik in der Anfangsphase der Weltwirtschaftskrise, München 1987, S. 173, erkennt richtig den Zweck einer potenziellen politischen Kontrolle kommerzieller Kreditströme. Hermann Graml, Zwischen Stresemann und Hitler. Die Außenpolitik der Präsidialkabinette Brüning, Papen und Schleicher, München 2001, S. 81, hat im Bericht die Idee, „kurzfristige Kredite direkt über die Regierungen zu leiten“, erkannt. Doch geht das wohl über das Gemeinte hinaus, wäre jedenfalls von deutscher Seite von vornherein undenkbar gewesen - nicht wegen nationalistischer Stimmen in Deutschland sondern wegen der hier bestehenden Wirtschaftsordnung, in der die Regierung nicht als Kreditvermittler vorgesehen war.

${ }^{15}$ Köppen, Brünings Spardiktat, S. 353.

${ }^{16}$ Vgl. Erlass von Staatssekretär von Bülow an Botschafter Hoesch vom 7. 8. 1930, in: ADAP, Bd. XV, Nr. 172, S. 430-437.
} 
von Kreditströmen nicht näher zu treten, ausdrücklich eine Fortsetzung der von Hoesch geführten Gespräche auch in Hinblick auf die von Hoesch ins Spiel gebrachten langfristigen Kredite für erwünscht gehalten. Dazu gleich mehr.

In seiner ausführlichen Analyse des hinsichtlich französischer Kredite an deutsche Schuldner seinerzeit Bekannten hat Bülow die von Tardieu genannte Zahl von $25 \mathrm{Mrd}$. als weit übertrieben bezeichnet. Auch hätten sich deutsche Bankiers bei einer Umfrage an einer Ausdehnung kurzfristigen Kredits aus Frankreich nicht interessiert gezeigt. „Bei der politischen Unsicherheit der französischen Kredite und bei der Ungewißheit, wie die Geldmärkte sich später gestalten werden, möchten die deutschen Bankiers aber das gegenwärtig bestehende Verhältnis der Kreditaufnahme in Frankreich, England, den Vereinigten Staaten und auf anderen Geldmärkten nicht in der Richtung einer stärkeren Engagierung in Frankreich verschieben. ${ }^{\text {"17 }}$ Dass diese Sache auch den Franzosen eigentlich nicht dringlich erschien, konnte kurze Zeit später Botschafter Hoesch aus Paris berichten. In einem Gespräch habe Finanzminister Reynaud anerkannt, „daß Frage kurzfristigen Kredits für uns nur von geringem Interesse sei und daß wir die Gewährung langfristiger Gelder anstreben müssen“18.

III.

Genau dies hatte Hoesch schon im Gespräch am 9. Juli vorgebracht (siehe den letzten Satz in seinem Telegramm), hierzu aber nur von „einer ziemlich zurückhaltenden Aufnahme“ berichten können. Es ist mir völlig unerklärlich, weshalb Köppen diesen Satz aus seiner Inhaltsangabe des Gesprächs fortgelassen hat. Er geht auch an keiner anderen Stelle seines - angeblich französische Kapitalangebote behandelnden - Aufsatzes darauf (und dann auch auf die sichtliche „Zurückhaltung") ein. Gewiss, es handelt sich offensichtlich um eine deutsche Kreditnachfrage, kein französisches Angebot. Aber wenn es zu diesem Zeitpunkt auf französischer Seite auch nur den Anflug einer Neigung gegeben haben sollte, entweder direkt langfristigen Kredit an Deutschland zu geben oder indirekt an seiner Aufbringung mitzuwirken, wäre das jetzt der geeignete Moment gewesen. Die von Hoesch am 9. Juli beschriebene „Zurückhaltung“ auf französischer Seite ist, wie nun noch kurz darzustellen ist, nie aufgegeben worden.

Die Aktenpublikationen enthalten zahlreiche Dokumente, in denen von Gesprächen zwischen der deutschen und der französischen Seite über Wirtschaftsfragen und vage auch über Kredite verschiedener Art berichtet wird. Ein deutsches Interesse an langfristigen Krediten ist mannigfach belegt ${ }^{19}$. Wiederholt hat die französische Seite auch Bereitschaft bekundet, über Langfristkredite an deut-

${ }^{17}$ Ebenda, S. 434. Wie gefährlich französische Kurzfristkredite tatsächlich waren, zeigten die erheblichen Abzüge nach der Septemberwahl.

18 Botschafter in Paris an Auswärtiges Amt, 13.8. 1930, in: ADAP, Bd. XV, Nr. 178, S. 447.

${ }^{19}$ Vgl. u.a. Berichte von Hoesch vom 23. 10. 1930 über ein Gespräch mit Berthelot und vom 26. Oktober über ein Gespräch mit Tardieu, in: ADAP, Bd. XVI, Nr. 17, S. 38f. und Nr. 22, S. $48 \mathrm{f}$. 
sche Unternehmen nachzudenken ${ }^{20}$. Aber nie ist vom Juli 1930 bis zum Abbruch derartiger Gespräche (Ankündigung des Plans einer deutsch-österreichischen Zollunion) von etwas die Rede, was man als Angebot eines Kredits an die Reichsregierung bezeichnen könnte und auf das die Reichsregierung nicht eingegangen wäre, gar, das sie abgelehnt hätte. Es hat eben, wie das schon 1987 Franz Knipping trefflich formuliert hat, „politisch motivierte[n] französische Stützungshemmungen“ gegeben ${ }^{21}$. Statt weiterer einzelner Gesprächs-Nachweise seien hier die Aussagen des besten Zeugen der deutsch-französischen Verhandlungen in der fraglichen Zeit zitiert, die Aufzeichnung von Botschafter Leopold von Hoesch vom 6. März 1931:

„Wenn die Erörterung sich dann den finanziellen Fragen zuwendet, so besteht zuerst Einhelligkeit in der Überzeugung, daß alles dahin drängt, den französischen Kapitalien den Weg zu langfristiger Anlage in Deutschland zu erschließen: Gold- und Geldüberfluß in Frankreich, niedriger Zinssatz daselbst, Gefahr der Geldinflation, verbunden mit Gefahr der Preissteigerung, andererseits Geldnot in Deutschland, hohe Zinssätze, große Kapitalertragsmöglichkeiten, politisches Interesse. Es entsteht zunächst begeisterte Übereinstimmung, bis die Frage praktisch gestellt wird. Deutscherseits wird die Ausgabe irgendeiner z. B. städtischen Anleihe vorgeschlagen, worauf französischerseits die Befürchtung ausgesprochen wird, das Publikum, das an der Younganleihe viel verloren habe und dem es an Vertrauen auf die Stabilität der innerdeutschen Verhältnisse fehle, werde deutsche Obligationen nicht zeichnen. Da andrerseits französische Banken kein Papier ausgeben, dessen Aufnahme durch das Publikum ihnen nicht gesichert erscheint, werden die Aussichten für die Auflegung einer deutschen Anleihe bald pessimistisch beurteilt. Auf die Frage, was denn geschehen müsse, um das französische Publikum zur langfristigen Investierung seines Geldbesitzes in Deutschland zu veranlassen, erfolgt, oft unter Hinweis auf die Sanierungsmethoden Poincarés, die allerseits als richtig erkannte Antwort, es müsse erst ,Vertrauen“ geschaffen werden. Bei der Erörterung der Frage, wie man Vertrauen schaffen könne, stößt man bald auf die Antwort, Vertrauen erheische eine vorhergehende restlose politische Verständigung, worauf man wieder am Ausgangspunkt der Unterhaltung angekommen ist, ohne bei diesem Kreislauf ein Ergebnis erzielt zu haben." 22

${ }^{20}$ Schon in seinem Gespräch mit von Hoesch erklärte der französische Finanzminister am 12. August, „er sei grundsätzlich mit Gedanken langfristiger Investierung französischen Kapitals in deutschen Werken einverstanden. [...] Freilich erheische eine Orientierung des französischen Kapitalmarktes nach Deutschland hin Vertrauen und Sicherheit, und zwar in erster Linie auf politischem Gebiet." ADAP, Bd. XV, Nr. 178, S. 447. Es blieb also auch hier bei der schon am 9. Juli von Tardieu vertretenen Linie.

${ }^{21}$ Knipping, Deutschland, Frankreich, S. 202.

${ }^{22}$ Botschafter in Paris von Hoesch an das Auswärtige Amt, Paris 6. 3. 1931, in: ADAP, Bd. XVII: 1. März bis 30. Juni 1931, Göttingen 1982, Nr. 3, S.9f. Graml, Zwischen Stresemann und Hitler, S.87, deutet Hoeschs realistische Einschätzung der Möglichkeiten als einen „Anfall von Pessimismus“, meint aber, bei gutem Willen von deutscher Seite wäre (ausreichender?) 
IV.

Doch ist ja nicht nur geredet worden. Für die von Köppen so betonte „Hilfsbereitschaft" Frankreichs gab es in der hier interessierenden Zeit einen praktischen Testfall, den Lee Higginson Kredit. Als sich im September/Oktober 1930 die Lage der Reichskasse neuerlich dramatisch verschlechterte und wiederum die Zahlungsunfähigkeit drohte, fand sich nach Verhandlungen, die schon im März 1930 begonnen und danach wiederholt unterbrochen worden sind, ein ausländisches Bankenkonsortium unter Führung des amerikanischen Bankhauses Lee, Higginson \& Co bereit, einen Überbrückungskredit in Höhe von 100 bis 125 Millionen \$ auf maximal 2 Jahre zu organisieren ${ }^{23}$. Von Seiten der internationalen Gläubiger und insbesondere auch in der deutschen Regierung wurde die Beteiligung französischer Banken, und sei es nur eine symbolische, ausdrücklich gewünscht ${ }^{24}$. Wie dringlich dieser Kredit in Hinblick auf die drohende Zahlungsunfähigkeit gewesen ist, erweist der Umstand, dass die deutsche Regierung nicht umhin kam, für diesen Kredit demütigende Bedingungen hochpolitischer Art zu akzeptieren, so die Einbringung und Verabschiedung eines Schuldentilgungsgesetzes im Reichstag sowie inhaltlich vorgegebene Beschlüsse der gesetzgebenden Organe hinsichtlich eines drastischen Haushaltssanierungs- also Sparprogramms (u.a. Erhöhung des Beitrags zur Arbeitslosenversicherung, Kürzung von Beamtengehältern sowie der Steuerüberweisungen des Reiches an die Länder), wie es dann in der Notverordnung vom 1. Dezember 1930 auch festgelegt worden ist ${ }^{25}$. Obgleich gerade die französische Seite derart harte Sanierungsmaßnahmen zur Bedingung einer Beteiligung an der Anleihe gemacht hatte, kam eine solche letztendlich nicht zustande. Die französische Regierung hat sich nämlich geweigert,

Kredit zu bekommen gewesen. - Eine ausführliche Analyse der Notwendigkeit langfristiger Auslandskredite wie auch der geringen Chancen, solche zu erlangen, findet sich in der Denkschrift des Ministerialrates Berger über die Reparationspolitik vom 2.4.1931, in: Ilse Maurer/Udo Wengst, Politik und Wirtschaft in der Krise 1930-1932. Quellen zur Ära Brüning, Erster Teil, Düsseldorf 1980, Nr. 204, S. 571-582, hier S.579f.

23 „Überbrückungskredit“ in Hinblick darauf, dass im Oktober 1929 eine langfristig laufende Anleihe mit dem Kreuger-Konzern vereinbart worden ist, die in zwei Tranchen nach Begebung der Young-Anleihe ausgezahlt werden sollte.

24 „Wenngleich eine französische Beteiligung an dem Kredit von 125 Millionen Dollar zahlenmäßig nur von geringer Bedeutung ist, ist auf die französische Beteiligung aus allgemeinpolitischen Gründen doch sehr großer Wert zu legen." Aufzeichnungen des Staatssekretärs in der Reichskanzlei Pünder über Anleiheverhandlungen, 6.10.1930, in: Akten der Reichskanzlei, Die Kabinette Brüning I und II, Bd. 1, Nr. 131, S. 502.

${ }^{25}$ Zur schweren Demütigung, die das für die Reichsregierung bedeutete, siehe Harold James, Deutschland in der Weltwirtschaftskrise 1924-1936, Stuttgart 1988, S. 82 f. Zum Lee Higginson-Kredit im Allgemeinen siehe James, The Reichsbank, S. 120-127; Knipping, Deutschland, Frankreich, S. 200; Gerhard Schulz, Zwischen Demokratie und Diktatur. Verfassungspolitik und Reichsreform in der Weimarer Republik, Bd. III: Von Brüning zu Hitler. Der Wandel des politischen Systems in Deutschland 1930-1933, Berlin u.a. 1992, S. 225-229; Philipp Heyde, Das Ende der Reparationen. Deutschland, Frankreich und der Youngplan 1929-1932, Paderborn 1998, S. 135-136; besonders ausführlich Bachmann, Reichskasse und öffentlicher Kredit, S. 211-222. 
auf den Wunsch des an den Kreditverhandlungen beteiligten Crédit Lyonnais einzugehen und die Beteiligung französischer Banken ausdrücklich zu befürworten. Einem Bankenvertreter gegenüber erklärte Ministerpräsident Tardieu, eine öffentliche Stellungnahme dieser Art würde seine wackelige Regierung zu Fall bringen ${ }^{26}$. So viel zum Spielraum der französischen Seite für finanzielle Freundlichkeiten gegenüber Deutschland in der zweiten Jahreshälfte $1930^{27}$.

Allerdings hat es Anfang 1931 dennoch eine Beteiligung französischer Banken an einem Kredit gegeben, der der Abwehr der Zahlungsunfähigkeit des Reiches dienen sollte. Sie ist sogar ausdrücklich von der französischen Regierung unterstützt worden. Köppen geht darauf nicht ein. Weil aber auch Thomas Ferguson und Peter Temin, zwei amerikanische Wirtschaftshistoriker, an die hierbei erstmals nicht nur beredete sondern praktizierte französische Konzessionsbereitschaft sehr weitgehende Folgerungen in Hinblick auf den möglicherweise zu verhindernden Fortgang der Geschichte geknüpft haben ${ }^{28}$, erscheint es mir nützlich, auch hier genauer hinzusehen ${ }^{29}$.

Als im Januar 1931 nach einer neuerlichen Schrumpfung der Einnahmen und wachsender Beanspruchung der Arbeitslosenversicherung erneut große Deckungslücken in der Reichskasse erkennbar wurden, für die im Inland keine ausreichende Finanzierung mehr zu finden war, kam man auf eine schon von Finanzminister Rudolf Hilferding (SPD) in der Mai-Krise 1929 ins Spiel gebrachte Idee zurück, nämlich den Verkauf von im Besitz des Reiches befindlichen Vorzugsaktien der Reichsbahn ${ }^{30}$. Der Verkauf von „Tafelsilber“ ist ja die schnellst mögliche Form zu Geld zu kommen. Als Käufer fand sich die Reichsversicherungsanstalt für Angestellte. Sie war zur Bildung eines Vermögensstocks verpflichtet. Allerdings konnte die Versicherungsanstalt nicht sofort, sondern erst später bezahlen. Um dem Reich den Verkaufserlös möglichst rasch zur Verfügung zu stellen, erklärte sich das Bankhaus Lee, Higginson \& Co. bereit, als Konsortialführer unter

${ }^{26}$ Siehe James, The Reichsbank, S. 126.

${ }^{27}$ Zur öffentlichen Meinung hinsichtlich der Beziehungen zu Deutschland vgl. neuerdings Franziska Brüning, Frankreich und Heinrich Brüning. Ein deutscher Kanzler in der französischen Wahrnehmung, Stuttgart 2012.

${ }^{28}$ Vgl. Thomas Ferguson/Peter Temin, Made in Germany: The German Currency Crisis of July, 1931, in: Research in Economic History, Bd. 21 (2003), S. 1-53; online: http://papers.ssrn. com/sol3/papers.cfm?abstract_id=260993 [letzter Zugriff: 8.1.2015]. Ihre These ist, dass bei einem größeren Entgegenkommen der deutschen Seite hinsichtlich von Frankreich geforderter politischer Garantien im Frühjahr 1931 Anleihen für das Reich zu bekommen waren und so der Tributaufruf vom 6. Juni 1931 und die Bankenkrise hätten vermieden werden können. Die Autoren behaupten nicht, Brüning hätte dank solcher Kredite seine restriktive Politik aufgeben und ein deficit spending betreiben können.

${ }^{29}$ Zum Vorgang vgl. Schulz, Zwischen Demokratie und Diktatur, S. 294-298; Bachmann, Reichskasse und öffentlicher Kredit, S. 235-242; Heyde, Das Ende der Reparationen, S. 141-143. Graml, Zwischen Stresemann und Hitler, S. 86, überrascht hingegen mit der Feststellung: „Auch in den ersten Monaten des Jahres 1931 blieb sichtbare Bewegung [in der Frage französischer Finanzhilfen] aus."

${ }^{30}$ Vgl. Hilferding-Vortrag in der Kabinettsitzung vom 3.5.1929, in: Akten der Reichskanzlei. Das Kabinett Müller II: 28. Juli 1928 bis 27. März 1930, Bd. 1, bearb. von Martin Vogt, Boppard 1970, Nr, 193, S. 628. 
erhofft breiter Beteiligung anderer ausländischer Banken, insbesondere französischer, der Reichsversicherungsanstalt den Kauf vorzufinanzieren. Schuldner dieses kurzfristigen Vorfinanzierungskredits ${ }^{31}$ war somit nicht das Reich sondern die Reichsversicherungsanstalt. Wie schon beim Überbrückungskredit vom Oktober 1930 war sowohl Lee, Higginson \& Co. als auch den anderen Banken wie der deutschen Regierung eine Beteiligung französischer Banken ausdrücklich sehr erwünscht. Kommerziell handelte es sich für die Kreditgeber angesichts der gegebenen Sicherheiten und des hohen Diskontsatzes von 6 1/2 Prozent um ein gutes Geschäft. Doch machten die interessierten französischen Banken auch hier ihre Beteiligung von einer förmlichen Ermutigung durch die Regierung abhängig. Zu sehr fürchteten sie angesichts der generellen Stimmung in Frankreich gegenüber Deutschland die Kritik des Publikums und darauf folgend eine Störung ihres innerfranzösischen Geschäfts. Erst als die erwünschte „lettre d'encouragement“ des Finanzministers vorlag, wurde der Kreditvertrag unterzeichnet ${ }^{32}$. Die französische Beteiligung war hinsichtlich ihrer Symbolwirkung gewiss eine bedeutsame Aktion. Aber dieser ohnehin schon sehr merkwürdige indirekte Kredit an das Reich löste natürlich nicht die weiterhin zu erwartenden Kassenprobleme der Regierung. Hat er aber ein Tor der Hoffnung geöffnet?

\section{V.}

Mit der offiziellen Ermutigung der Banken hat sich die französische Regierung erhebliche innenpolitische Probleme eingehandelt. Angesichts der Notwendigkeit für die Regierung, sich gegen die erwartete Kritik in der Kammer verteidigen zu müssen, ist im Finanz- und Außenministerium ein außerordentlich interessanter Plan zur stufenweisen Entfaltung finanzieller Hilfen für Deutschland entwickelt worden. Er ist noch vor der beabsichtigten Beschlussfassung im Kabinett am 14. März vom Generalsekretär des Außenministeriums Berthelot dem deutschen Botschafter bekanntgegeben und sogar auszugsweise vorgelesen worden. Wegen der Spekulationen von Paul Köppen, Thomas Ferguson und Peter Temin und wegen einiger Missverständnisse in der Literatur hinsichtlich der ökonomischen Bedeutung für wahrscheinlich gehaltener französischen Finanzhilfen erscheint es nützlich, das vom Botschafter hierzu Festgehaltene im Wortlaut zu zitieren $^{33}$ :

${ }^{31}$ Unter Berufung auf ihre Quelle, die New York Times vom 6.2.1931, erwecken Ferguson/ Temin, Made in Germany, den falschen Eindruck, es habe sich um einen langfristigen Kredit gehandelt. Tatsächlich begann die ratenweise Rückzahlung schon - rückdatiert - im Januar 1931.

${ }^{32}$ Der Gesamtumfang betrug 32 Millionen \$ (ca. 135 Millionen RM), französische Banken waren mit 10 Millionen $\$$ beteiligt.

${ }^{33}$ ADAP, Bd. XVII, Dok.15, Botschafter von Hoesch an das Auswärtige Amt, 14.3.1931, S. 42. Zwar handelt es sich bei diesem Plan erst um eine vom Direktor der Unterabteilung für Handelsbeziehungen im französischen Außenministerium Coulondre konzipierte Vorlage, aber die Tatsache, dass der Generalsekretär des Ministeriums sie vertraulich dem Botschafter vorliest und in Sachen Kreditgeschäfte keine präziseren und weitergehenden Vorstellungen 
„Es würde wirkungslos sein und dem verfolgten Ziel nicht dienen, wenn Frankreich sich auf ungeregelte und systemlose Finanztransaktionen von Fall zu Fall beschränken wollte. Vielmehr müsse nach einem bestimmten Plan vorgegangen werden, der sich etwa in folgenden Etappen abwickeln könne:

1.) Inangriffnahme des Gedankens des Ausbaues der deutschen Sachlieferungen in größerem Stil,

2.) Vermehrung der Deutschland zur Verfügung gestellten kurzfristigen Gelder mit der Tendenz, die entstehenden Verpflichtungen nach und nach in langfristige umzuwandeln,

3.) Zulassung deutscher Wertpapiere zum Handeln [sic!] an den französischen Börsen,

4.) Auflegung deutscher Anleihen in Frankreich, und zwar erstlinig hypothekarisch gesicherte Kredite und Stadtanleihen. Zu diesem Punkt 4) wird bemerkt, daß es zu seiner Verwirklichung natürlich einer Vorbereitung und der Schaffung einer Atmosphäre des Vertrauens bedürfe.“

Die einzelnen Etappen wären sicherlich in Hinblick auf die deutsch-französischen Beziehungen interessante Verhandlungsgegenstände gewesen. Aber schon der erste Schritt hätte doch wohl erhebliche Zeit in Anspruch genommen ${ }^{34}$. Selbst mit großem Wohlwollen lässt sich aus diesem Plan kein Geld- oder Kapitalangebot konstruieren, welches zur Lösung der Haushaltsprobleme Brünings hätte beitragen können. Deshalb hat auch die Offenlegung der deutsch-österreichischen Zollunionspläne am 17. März 1931, die das bilaterale Klima wieder dramatisch verschlechterte, keine wohl begründete Hoffnung auf rasche finanzielle Hilfe aus Frankreich zerstören können.

Sollte Deutschland nicht zahlungsunfähig werden, mussten die Ausgaben des Reiches den ständig sinkenden Einnahmen angepasst werden. Dass dies nicht eine spezielle Idee von Brüning gewesen ist, ergibt sich schon daraus, dass bereits die Vorgängerregierung zu einem Schwenk in eine restriktive Fiskalpolitik gezwungen war ${ }^{35}$. Nach ersten Ansätzen ist die Regierung Müller bekanntlich an dieser Aufgabe gescheitert und hat sie ihrer Nachfolgerin weiter gereicht.

Möglicherweise führen die Erfahrungen der letzten Jahre mit der anhaltenden Krise in einigen südeuropäischen Staaten zu einem größeren Verständnis dafür, dass die Sanierung von Staatshaushalten in parlamentarischen Verfahren an zahlreiche innen- und außenpolitische Voraussetzungen geknüpft ist, die - jedenfalls in Deutschland 1929/30 - nicht hinreichend gegeben waren.

bekannt sind, erscheint die Vermutung nicht unbegründet, dass dies den augenblicklichen Stand der günstigsten „Angebote“ der Regierung darstellen dürfte.

${ }^{34}$ Hoesch meinte in einem Telefongespräch mit Staatssekretär Bülow, es ginge hier nicht um die 90 Millionen notleidende Reparations-Sachlieferungen sondern um Projekte, „die noch keine Gestalt angenommen hätten“, in: ADAP, Bd. XVII, Fußnote zu Nr. 15, S. 43.

${ }^{35}$ Vgl. Ilse Maurer, Reichsfinanzen und große Koalition. Zur Geschichte des Reichskabinetts Müller (1928-1930), Bern/Frankfurt a.M. 1973, und die in Anmerkung 2 genannten Veröffentlichungen. 


\section{DE DE GRUYTER}

\section{FESTSCHRIFT ZU VOLKER SELLLINS 75. GEBURTSTAG}

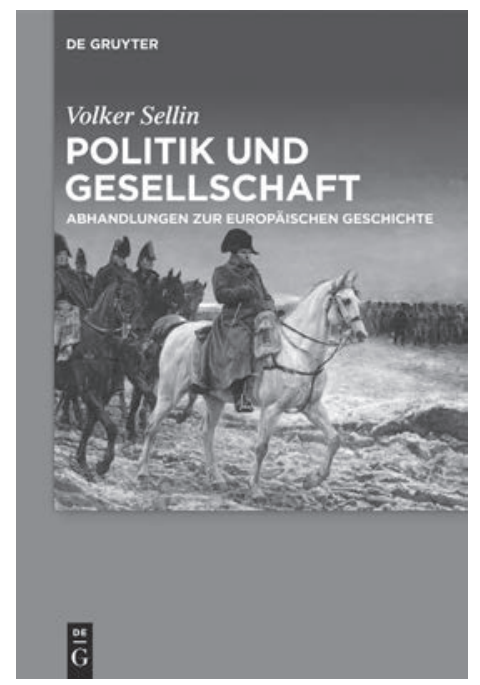

Volker Sellin

Politik und Gesellschaft

Abhandlungen zur europäischen Geschichte

Mit einem Vorwort des Herausgebers Frank-Lothar Kroll

2015, X, 567 S., 15 Abb.

Ln. $€ 89,95$

ISBN 978-3-11-036335-7

eBook $€ 89,95$

PDF ISBN 978-3-11-036348-7

ePUB ISBN 978-3-11-039860-1

Print +eBook $€ 139,95$

ISBN 978-3-11-036349-4

Der Band vereinigt 26 ausgewählte, zum Teil noch unveröffentlichte Abhandlungen des Heidelberger Historikers Volker Sellin zur europäischen Geschichte der Neuzeit. Am Anfang steht ein Aufsatz zur Mentalitätsgeschichte, der stark in die Forschung hineingewirkt hat. Thematische Schwerpunkte der folgenden Abhandlungen sind Legitimität und Scheitern von Monarchien und das Verhältnis von Monarchie und Nationalstaat. Mehrere Beiträge widmen sich dem Sturz Napoleons, seinem Tod und seinem Nachleben. Zwei weitere gewichtige Untersuchungen behandeln die Geschichte der Juden Italiens im Risorgimento und unter dem Faschismus. Abhandlungen zur Geschichte von Schloss und Universität Heidelberg beschließen den Band.

Volker Sellin ist Professor em. für Neuere Geschichte an der Universität Heidelberg. 